Published in final edited form as:

Eur Urol. 2016 September ; 70(3): 539-540. doi:10.1016/j.eururo.2016.06.029.

\title{
Re: Effects of Testosterone Treatment in Older Men
}

\author{
aDepartment of Urology, University of Miami Miller School of Medicine, Miami, FL, USA \\ Department of Urology, Baylor College of Medicine, Houston, TX, USA
}

\begin{abstract}
In the February issue of The New England Journal of Medicine, Snyder and colleagues [1] trials are set of primarily at determinin bone density, anemia, and cardiovascular function were not reported in this initial manuscript.
\end{abstract}

Seven hundred and ninety men over the age of $65 \mathrm{yr}$ with symptoms of hypogonadism and a serum testosterone concentration of $<275 \mathrm{ng} / \mathrm{dl}$ were randomized to receive either an initial exual activity (an improvement of $\geq 4$ in the Functional Assessment of Chronic Illness Therapy fatigue score). In the physical function trial, there was no significant difference between TST and placebo in terms of the percentage of men with an increase of $50 \mathrm{~m}$ or greater in the 6-min men diagnosed with prostate cancer $1 \mathrm{yr}$ after the study period. Similarly, there was no during the study period.

\footnotetext{
*Corresponding author. 1120 North West $14^{\text {th }}$ Street, Suite 1560, Miami, FL 33136, USA. ramasamy@ miami.edu (R. Ramasamy). Conflicts of interest: Ranjith Ramasamy is a clinical trials participant for Beckman Coulter and a member of advisory board for Lipocine Inc. Samarpit Rai, Jason Scovell, and Joseph Palmer have nothing to disclose.
} 


\section{Experts' comments}

The Testosterone Trials were designed to address the lack of definitive evidence [2-4] and controversy surrounding the risks and benefits of TST. The coordinated trials focused on symptoms that have been associated with low testosterone levels. The seven trials share similar inclusion criteria and methodologies that allow investigators to draw conclusions across all studies. The initial study report focusing on the three main trials (sexual function, physical function, and vitality) represents the most rigorously and appropriately designed study in the field of TST.

While the authors failed to provide the range of total testosterone levels at each measurement period despite collecting this data, they report that $91 \%$ of men maintained eugonadal levels throughout all time points during the study suggesting that therapy was effective in restoring testosterone levels.

The sexual function study identified that TST plays a positive role in improving sexual desire, frequency, and erectile function. Despite these improvements, the benefits are modest and may not be clinically meaningful. It is well described that an improvement of 4 or greater on the International Index of Erectile Function-Erectile Function is clinically relevant and is often used as the cut-off for clinical trials assessing erectile function. While many hoped that The Testosterone Trials would clearly prove or disprove the efficacy of TST, it is not surprising given the modest and mixed findings of previous smaller studies that the ultimate effect of TST on hypogonadal symptoms is mild. It is likely that in most patients TST has the capacity to improve sexual function, but this effect may not be sufficient as monotherapy.

There was no difference in the number of major cardiovascular events in men treated with TST or placebo. While the benefits of TST appear to be mild in this population, the risks appear to be even smaller. A small proportion of men had an increase in prostate specific antigen, although the study period was too short to identify de-novo prostate malignancy. We hope that long-term follow-up of these patients will be similarly reassuring.

After screening $>50000$ men, only $790(<2 \%)$ of them were found eligible to be enrolled in the trial. The average participant was aged $72 \mathrm{yr}$ and many men had comorbidities including a high rate of obesity, hypertension, sleep apnea, and diabetes. The strict inclusion criteria enhanced the study's ability to identify treatment efficacy. Because participation was limited to this narrow population, it is important not to generalize these findings to a larger population. While the majority of the controversy in TST has been focused on the older male population due to concerns over adverse events, there is a lack of high-quality evidence guiding therapy in younger men with hypogonadism.

This landmark study and future results that will be subsequently published will contribute significantly in shared decision making about the role of TST in older men with low testosterone levels. 


\section{References}

1. Snyder PJ, Bhasin S, Cunningham GR, et al. Effects of testosterone treatment in older men. N Engl J Med. 2016; 374:611-24. [PubMed: 26886521]

2. Liverman, CT.; Blazer, DG. Testosterone and aging: Clinical research directions. Washington, DC: National Academies Press; 2004.

3. Srinivas-Shankar U, Roberts SA, Connolly MJ, et al. Effects of testosterone on muscle strength, physical function, body composition, and quality of life in intermediate-frail and frail elderly men: A randomized, double-blind, placebo-controlled study. J Clin Endocrinol Metab. 2010; 95:639-50. [PubMed: 20061435]

4. Basaria S, Coviello AD, Travison TG, et al. Adverse events associated with testosterone administration. N Engl J Med. 2010; 363:109-22. [PubMed: 20592293] 